\title{
Disordered chaotic strings
}

\section{Mirko Schäfer and Martin Greiner}





\title{
Disordered chaotic strings
}

\author{
Mirko Schäfer ${ }^{1}$ and Martin Greiner ${ }^{2}$ \\ ${ }^{1}$ Frankfurt Institute for Advanced Studies, \\ Johann Wolfgang Goethe University, Frankfurt, Germany \\ ${ }^{2}$ Aarhus School of Engineering and Department of Mathematical Sciences, \\ Aarhus University, Aarhus, Denmark
}

\begin{abstract}
Chaotic strings are coupled Tchebyscheff maps on a ring-network. With a wellspecified empirical prescription they are able to explain the coupling constants of the standard model of elementary particle physics. This empirical relationship is tested further by introducing a tunable disorder to chaotic strings. Inhomogeneous coupling weights as well as small-world perturbations of the ring-network structure are discussed. It is found that certain combinations of coupling and network disorder preserve the empirical relationship between chaotic strings and the weak and strong sector of the standard model of elementary particle physics. For the electromagnetic sector it is found that already a small disorder pushes the associated energy scale of the running coupling constant far away from the result without disorder.
\end{abstract}

Keywords: coupled map lattice, disorder phenomena, complex networks, standard model of elementary physics

\section{Introduction}

Since their introduction in the 80's by Kaneko, coupled map lattices have been widely studied as models of various spatiotemporal phenomena in extended systems $[1,2,3,4,5,6]$. Recently the investigations have been extended to coupled chaotic map networks, with particular interest in synchronization properties $[7,8,9,10,11$, $12,13]$.

A different application of coupled map lattices has been presented in $[14,15$, 16], where coupled Tchebyscheff maps on a 1-dimensional lattice were introduced as a model of vacuum fluctuations. The main result of this model, called chaotic strings, is a high-precision prediction of the numerical values of various coupling constants, masses and mixing angles of the standard model of elementary particle physics [17, 18]. Various physical embeddings for different parts of the models have been suggested $[14,15,16]$, but as of now it has not become clear which of these interpretations is correct, if any.

Corresponding author: Mirko Schäfer, schaefer@fias.uni-frankfurt.de 
In this paper we are interested in several extensions of the chaotic string model in itself and how these modifications influence the empirical relationship to the standard model of elementary particle physics. All extensions have to do with disorder phenomena. The first extension is coupling disorder and addresses the local randomization of the coupling weights of the Tchebyscheff maps. The second extension is network disorder and addresses the random small-world deformation of the ring topology.

\section{Chaotic strings and the standard model of elementary particles}

Coupled chaotic map networks are defined by the evolution equation

$$
x_{i}(t+1)=\left(1-\epsilon_{i}\right) f\left[x_{i}(t)\right]+\sum_{j=1}^{N} \frac{A_{i j} \epsilon_{i j}}{k_{i}} g\left[x_{j}(t)\right],
$$

with $x_{i}(t) \in[-1,1]$ associated to vertex $i$, the coupling weight $\epsilon_{i j} \in[0,1]$ between vertices $i$ and $j$, and

$$
\epsilon_{i}=\frac{1}{k_{i}} \sum_{j=1}^{N} A_{i j} \epsilon_{i j}
$$

The adjacency matrix $A_{i j}$ represents the network structure and determines the vertex degrees $k_{i}=\sum_{j=1}^{N} A_{i j}$. In [15] the network structure has been set to a 1dimensional lattice with periodic boundary conditions, that is a ring-network, which fixes $k_{i}=2$, and homogeneous coupling $\epsilon_{i j}=\epsilon_{i}=\epsilon$. The maps of interest for chaotic strings are Tchebyscheff polynomials up to third order in different combinations and are summarized in table 1.

Table 1: The six coupled map dynamics considered for chaotic strings [15].

\begin{tabular}{llc}
\hline label & \multicolumn{1}{c}{$f(x)$} & $g(x)$ \\
\hline $2 A$ & $T_{2}(x)=2 x^{2}-1$ & $T_{2}(x)=2 x^{2}-1$ \\
$2 B$ & $T_{2}(x)=2 x^{2}-1$ & $T_{1}(x)=x$ \\
$2 A^{-}$ & $T_{2}(x)=2 x^{2}-1$ & $T_{-2}(x)=-\left(2 x^{2}-1\right)$ \\
$2 B^{-}$ & $T_{2}(x)=2 x^{2}-1$ & $T_{-1}(x)=-x$ \\
$3 A$ & $T_{3}(x)=4 x^{3}-3 x$ & $T_{3}(x)=4 x^{3}-3 x$ \\
$3 B$ & $T_{3}(x)=4 x^{3}-3 x$ & $T_{1}(x)=x$ \\
\hline
\end{tabular}

The correlation function

$$
\begin{aligned}
W(\epsilon) & =\left\langle x_{i} x_{j}\right\rangle \\
& =\frac{1}{|\mathcal{E}|} \sum_{i<j} A_{i j} \frac{1}{T} \sum_{t=T_{0}+1}^{T_{0}+T} x_{i}(t) x_{j}(t)
\end{aligned}
$$




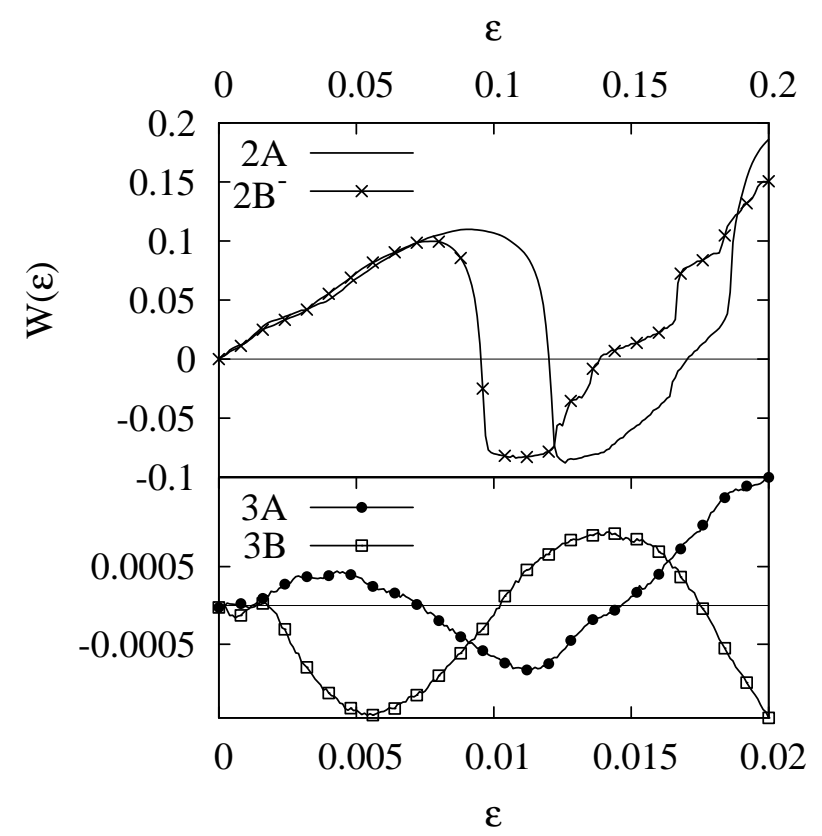

Figure 1: Dependence of the interaction energy $W(\epsilon)$ on the coupling parameter $\epsilon$ for some of the chaotic string dynamics considered in [15]. Top: $2 A$ (solid line) and $2 B^{-}$ (crosses) dynamics in the coupling region $\epsilon \in[0,0.2]$. Bottom: $3 A$ (closed circles) and $3 B$ (open squares) dynamics in the coupling region $\epsilon \in[0,0.02]$. The expression (2.3) has been calculated with a ring size $N=1001, T=10^{5}$ iterations, and an average over 10 different initial conditions.

between neighbouring nodes is interpreted as an interaction energy. The temporal average is taken over long iteration times $T$, excluding an initial transient $T_{0} . \mathcal{E}$ and $|\mathcal{E}|$ represent the set and the number of all edges, respectively. The dependence of the interaction energy on the coupling weight $\epsilon$ for some of the chaotic string dynamics is shown in Fig. 1. According to $[14,15]$, the coupling values $\epsilon^{*}$ leading to a vanishing interaction energy $W\left(\epsilon^{*}\right)=0$ with $d W(\epsilon) / d \epsilon<0$ are special. A surprising coincidence of these zeros with coupling constants of the standard model of elementary particle physics was reported. This is summarized in table 2 . The left side displays the zeros of the interaction energy for the chaotic string dynamics. The accuracy of the calculation only depends on the number of iterations. The right side of the table lists corresponding running standard model couplings. The indices denote the kind of interaction (electromagnetic, weak, strong) and particles (electron, up-, down-, top-quark, $W$-boson, Higgs boson, glueballs) involved. The energy scales at which the running couplings are evaluated are determined by the respective masses times some empirical prefactor; see [15] for more details.

Due to the unknown Higgs boson and glueball masses, a direct comparision of the last three cases in table 2 is not possible. In case of the Higgs boson, the comparison can be turned around to make a mass prediction $m_{H} \approx 154 \mathrm{GeV}$ [15], which agrees with the current upper experimental bound [19]. In Ref. [20] it has been shown that in the case of the two zeros connected to glueballs, the dynamics is non-ergodic and thus contradicts an interpretation as a model for vacuum fluctuations. As a 
Table 2: Zeros $\epsilon^{*}$ of the interaction energy (2.3) resulting from the coupled map string dynamics (2.1) listed in table 1, and the corresponding standard model (SM) couplings (taken from [15]).

\begin{tabular}{rr}
\hline zero & running SM coupling \\
\hline$\epsilon_{1}^{(3 A)}=0.0008164$ & $\alpha_{e l}^{d}\left(3 m_{d}\right) \approx 0.0008166$ \\
$\epsilon_{2}^{(3 A)}=0.0073038$ & $\alpha_{\text {el }}^{e}\left(3 m_{e}\right) \approx 0.007303$ \\
$\epsilon_{1}^{(3 B)}=0.0018012$ & $\alpha_{\text {weak }}^{u_{R}}\left(3 m_{u}\right)+\alpha_{\text {el }}^{d}\left(3 m_{d}\right) \approx 0.0018$ \\
$\epsilon_{2}^{(3 B)}=0.017550$ & $\alpha_{\text {weak }}^{\nu_{L}}\left(3 m_{d}\right)+\alpha_{e l}^{e}\left(3 m_{e}\right) \approx 0.01755$ \\
$\epsilon_{1}^{(2 A)}=0.120093$ & $\alpha_{s}\left(m_{W}+2 m_{d}\right) \approx 0.1208$ \\
$\epsilon_{1}^{(2 B)}=0.3145$ & $\alpha_{s}\left(m_{g g^{0++}}+2 m_{u}\right)$ \\
$\epsilon_{1}^{\left(2 A^{-}\right)}=0.1758$ & $\alpha_{s}\left(m_{g g^{2++}}+2 m_{b}\right)$ \\
$\epsilon_{1}^{\left(2 B^{-}\right)}=0.095370$ & $\alpha_{s}\left(m_{H}+2 m_{t}\right)$ \\
\hline
\end{tabular}

consequence, these two zeros have been ruled out as being connected to standard model couplings.

Given these results, the empirical relationship between the chaotic string model and the standard model of elementary particle physics is striking. Some physical embeddings for different parts of the chaotic string model have been suggested [14, $15,16]$. This of course deserves more clarification, but we will now follow a different perspective. The chaotic string model makes several assumptions, two of them being a uniform coupling weight and a ring-like network structure. We will extend the chaotic string model to include perturbations to the uniform coupling and the ring structure, and test their impact on the empirical relationship to the standard model of elementary particle physics.

\section{Coupling disorder}

A straightforward way to introduce perturbations to a uniform coupling is random disorder. It allows different coupling weights $\epsilon_{i j}$ between different neighbouring vertices. In order to assure that $x_{i}(t)$ is bound to $[-1,1]$, some care has to be taken. For every edge a random disorder number $\xi_{i j}$ is drawn from the uniform distribution defined on $[1-\Delta, 1+\Delta]$ with $\Delta \in[0,1]$. The disordered coupling weight $\epsilon_{i j}$ is then defined as

$$
\epsilon_{i j}=\frac{k_{i} \xi_{i j}}{\sum_{j=1}^{N} A_{i j} \xi_{i j}} \epsilon .
$$

The coupling weights $\epsilon_{i j}$ obtained by this procedure are symmetrically distributed around the average value $\epsilon$.

The interaction energy $W(\epsilon, \Delta)$ has now also become a function of the coupling disorder $\Delta$. The zeros of table 2 will shift as a function of $\Delta$. This is illustrated in Fig. 2. Our numerical calculations reveal that the zeros $\epsilon_{2}^{(3 B)}, \epsilon_{1}^{(2 A)}$ and $\epsilon_{1}^{\left(2 B^{-}\right)}$are shifted to smaller coupling values for increasing disorder parameter $\Delta$, whereas the zero $\epsilon_{2}^{(3 A)}$ is shifted to larger coupling values. The zeros $\epsilon_{1}^{(3 A)}$ and $\epsilon_{1}^{(3 B)}$ have not been 


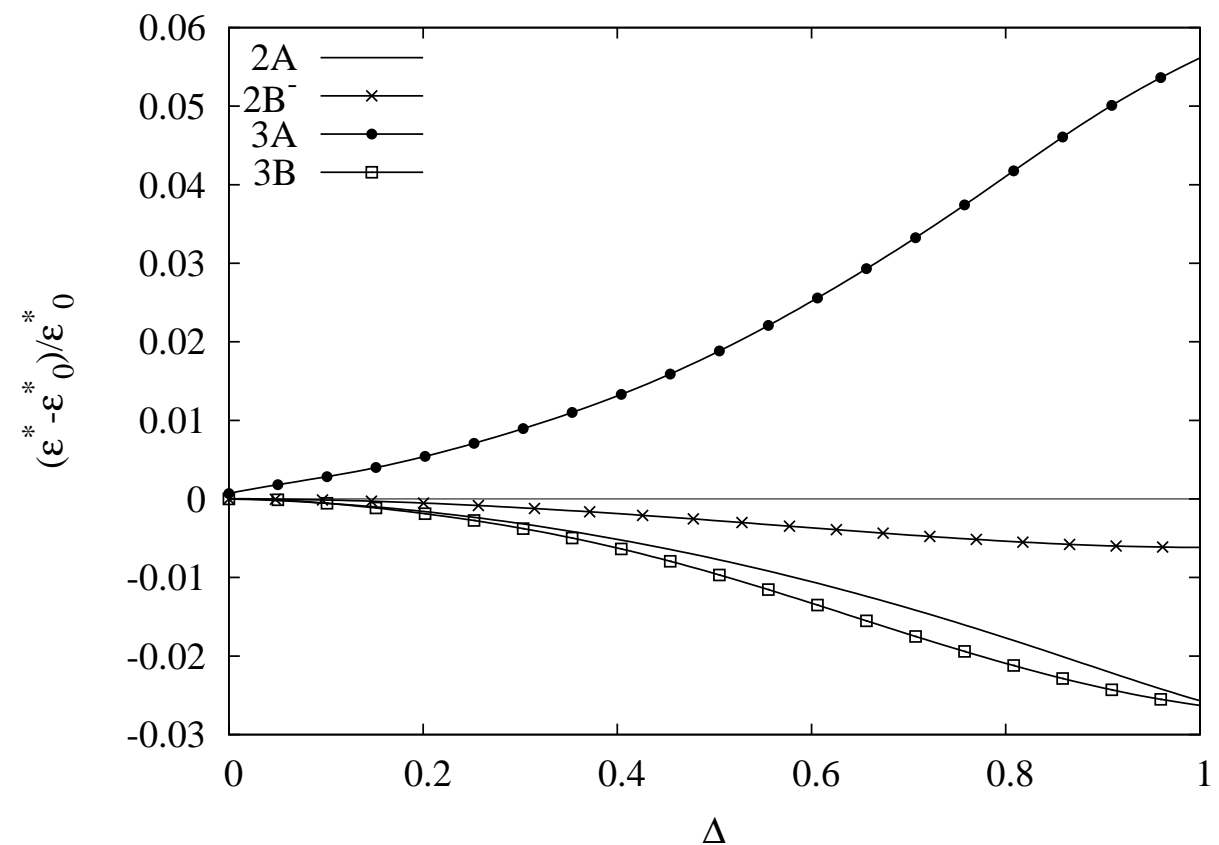

Figure 2: Relative shift $\left(\epsilon^{*}(\Delta)-\epsilon_{0}^{*}\right) / \epsilon_{0}^{*}$ of the zeros $\epsilon_{1}^{(2 A)}$ (solid line), $\epsilon_{1}^{\left(2 B^{-}\right)}$(crosses), $\epsilon_{2}^{(3 A)}$ (closed circles), $\epsilon_{2}^{(3 B)}$ (open squares) as a function of the coupling disorder $\Delta$. $\epsilon_{0}^{*}$ denotes the respective zero for zero disorder $\Delta=0$. The interaction energy has been calculated with a ring size $N=1001, T=10^{6}\left(2 A\right.$ and $2 B^{-}$dynamics $)$and $T=10^{7}(3 A$ and $3 B$ dynamics) iterations, and an average over 10 disorder realizations, respectively.

considered. Due to their smallness the numerical calculations are too expensive to reach acceptable convergence.

\section{Network disorder}

Network disorder is different to coupling disorder. The ring-network structure is perturbed and the coupling is kept homogeneous. A straightforward way to introduce perturbations to the ring-structure is to add random short-cuts, thus obtaining a small world network $[21,22]$. We start with the original 1-dimensional lattice, where every vertex $i$ is only coupled to its respective neighbours on each side, and thus has degree $k_{i}=2$, leading to an average degree $\langle k\rangle=2$ of the network. In order to obtain a small world network, additional edges $\mathcal{E}_{\text {add }}$ are randomly placed between previously uncoupled vertices. Since every new edge increases the degree of both adjacent vertices, the average degree of the resulting small world network is given by $\langle k\rangle=2+\left(2\left|\mathcal{E}_{\text {add }}\right| / N\right)$, which in the following is used as the disorder parameter.

The interaction energy $W(\epsilon,\langle k\rangle)$ is now also a function of the network disorder parameter. Its zeros will shift as a function of $\langle k\rangle$. This is illustrated in Fig. 3. Our numerical calculations reveal that for increasing network disorder all zeros are shifted to larger coupling values. 


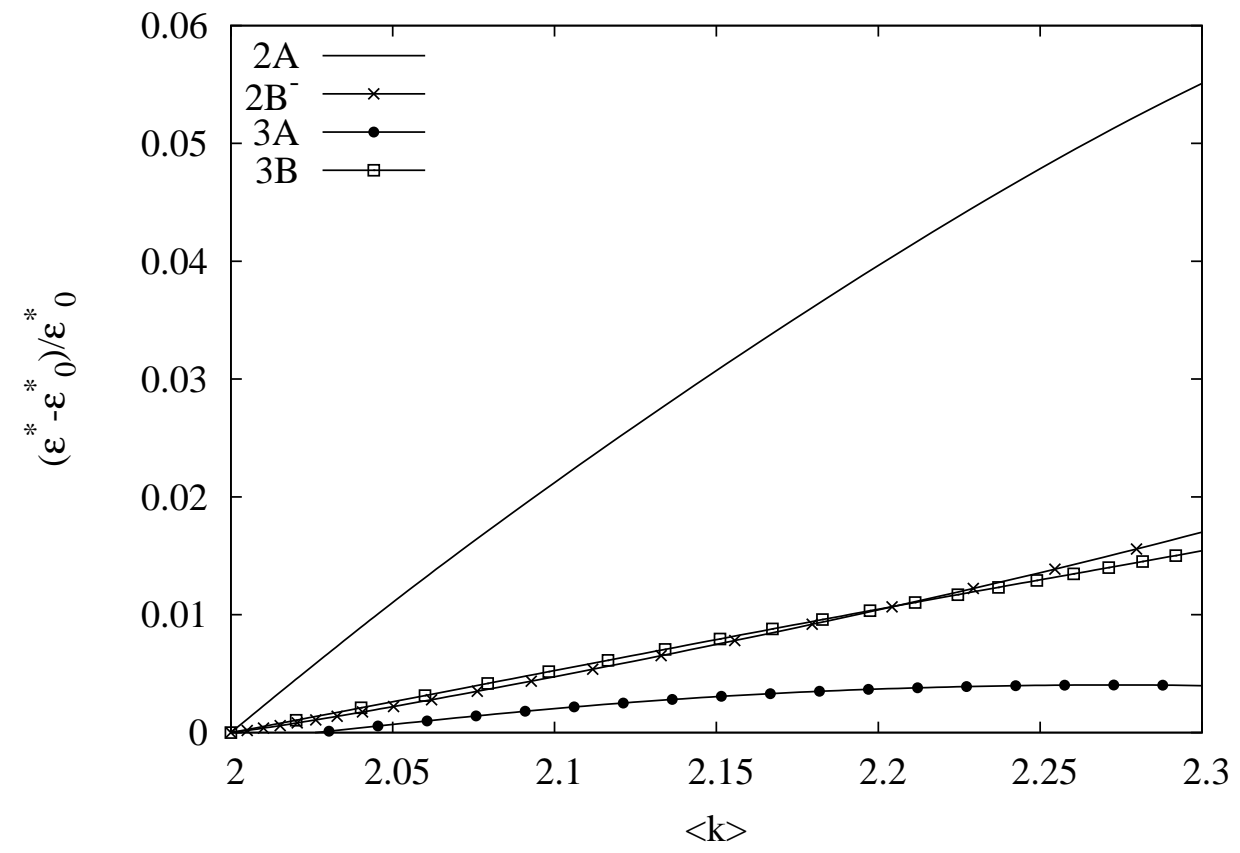

Figure 3: Relative shift $\left(\epsilon^{*}(\langle k\rangle)-\epsilon_{0}^{*}\right) / \epsilon_{0}^{*}$ of the zeros $\epsilon_{1}^{(2 A)}$ (solid line), $\epsilon_{1}^{\left(2 B^{-}\right)}$(crosses), $\epsilon_{2}^{(3 A)}$ (closed circles), $\epsilon_{2}^{(3 B)}$ (open squares) as a function of the network disorder $\langle k\rangle . \epsilon_{0}^{*}$ denotes the respective zero for zero disorder, that is a ring structure with $\langle k\rangle=2$. The interaction energy has been calculated with a ring size $N=1001, T=10^{6}$ iterations $(2 \mathrm{~A}$ and $2 B^{-}$dynamics), $T=10^{7}$ iterations ( $3 A$ and $3 B$ dynamics) and 10 network realizations.

\section{Discussion}

The $2 A$ and $2 B^{-}$couplings are associated with strong coupling constants $\alpha_{s}(E)$ at certain energy scales. A shift of the zeros $\epsilon^{*}=\alpha_{s}(E)$ thus can be related to a shift of the associated energy scale $E$. Figure 4 shows this energy shift for the $2 B^{-}$ dynamics.

According to [15], the zero $\epsilon_{1}^{\left(2 B^{-}\right)}$is associated with the strong coupling constant at an energy scale given by $E=2 m_{t}+E^{\prime}$, with $m_{t}$ the mass of the top quark and $E^{\prime}$ proposed to be the Higgs mass $m_{H}$. For zero coupling disorder and a ring-network one obtains $m_{H} \approx 160 \mathrm{GeV}$. ${ }^{1}$ This value is just above the currently estimated energy range $115 \mathrm{GeV}-150 \mathrm{GeV}$ of the Higgs mass[19]. In case of an experimental detection of the Higgs boson, the introduction of network disorder could then allow to shift the energy scale $E^{\prime}$ to the observed value, and thus to keep the interpretation $E^{\prime}=m_{H}$.

The $3 B$ zero is supposed to be related to a combination of two electroweak couplings. Hence a shift can be associated to a different combination or a change in the energy scale. Note however, that the energy-dependence of the electroweak coupling is much smaller than for the strong coupling.

As one can see in Figs. 2, 3, for three out of the four considered zeros the coupling and network disorder shift the zero $\epsilon^{*}$ in different directions. For these zeros we find

\footnotetext{
${ }^{1}$ Numerical differences to the result given in [15] arise from the way how the quark masses and thresholds are handled in the calculation of the running strong coupling constant.
} 


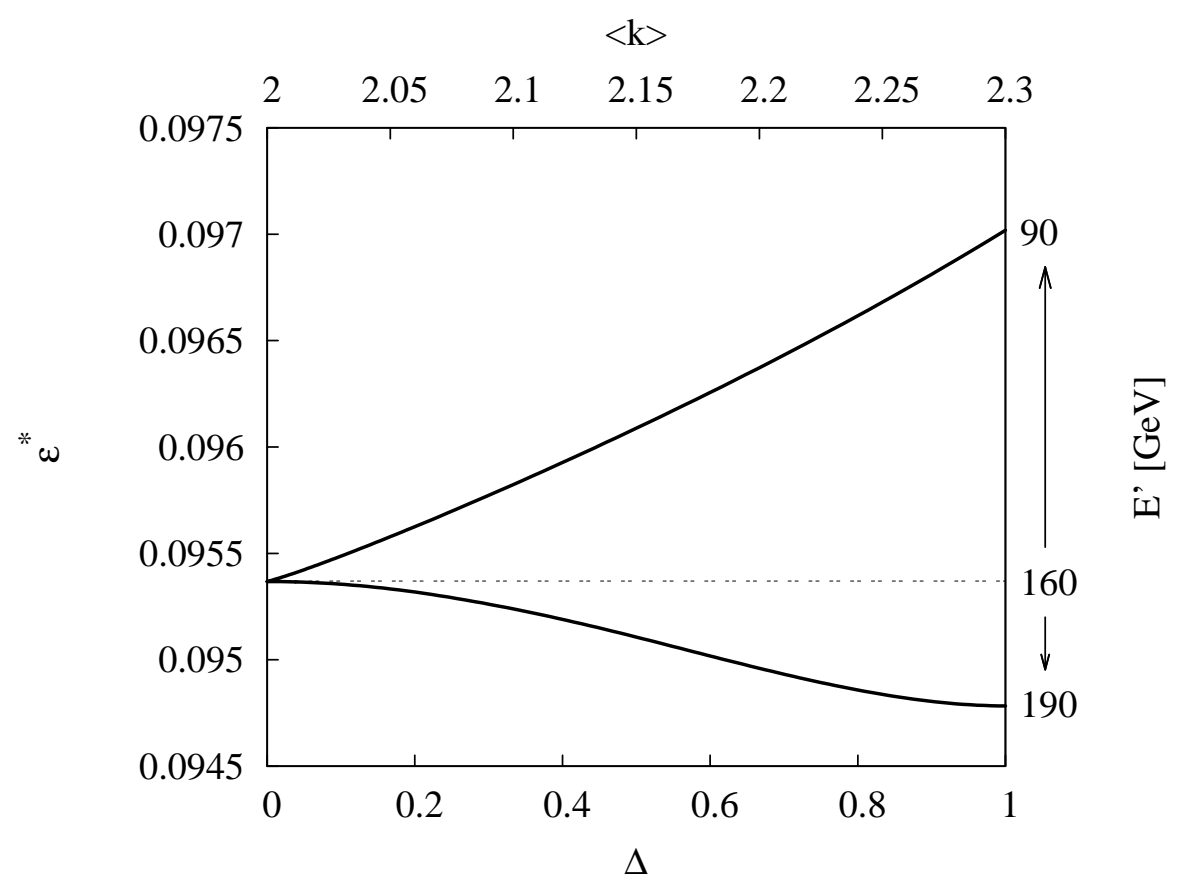

Figure 4: Energy shift in the interpretation of the zero $\epsilon_{1}^{\left(2 B^{-}\right)}$as a running strong coupling constant $\alpha_{s}(E)$. According to [15] the energy is given by $E=E^{\prime}+2 m_{t}$, where $m_{t}$ is the top quark mass (literature value $m_{t}=170.9 \mathrm{GeV}[18]$ ), and $E^{\prime}$ is supposed to be the Higgs mass $m_{H}$. The lower part shows the shift due to coupling disorder, whereas the upper part shows the shift due to network disorder. The running strong coupling $\alpha_{s}(E)$ has been calculated as in [23]. 


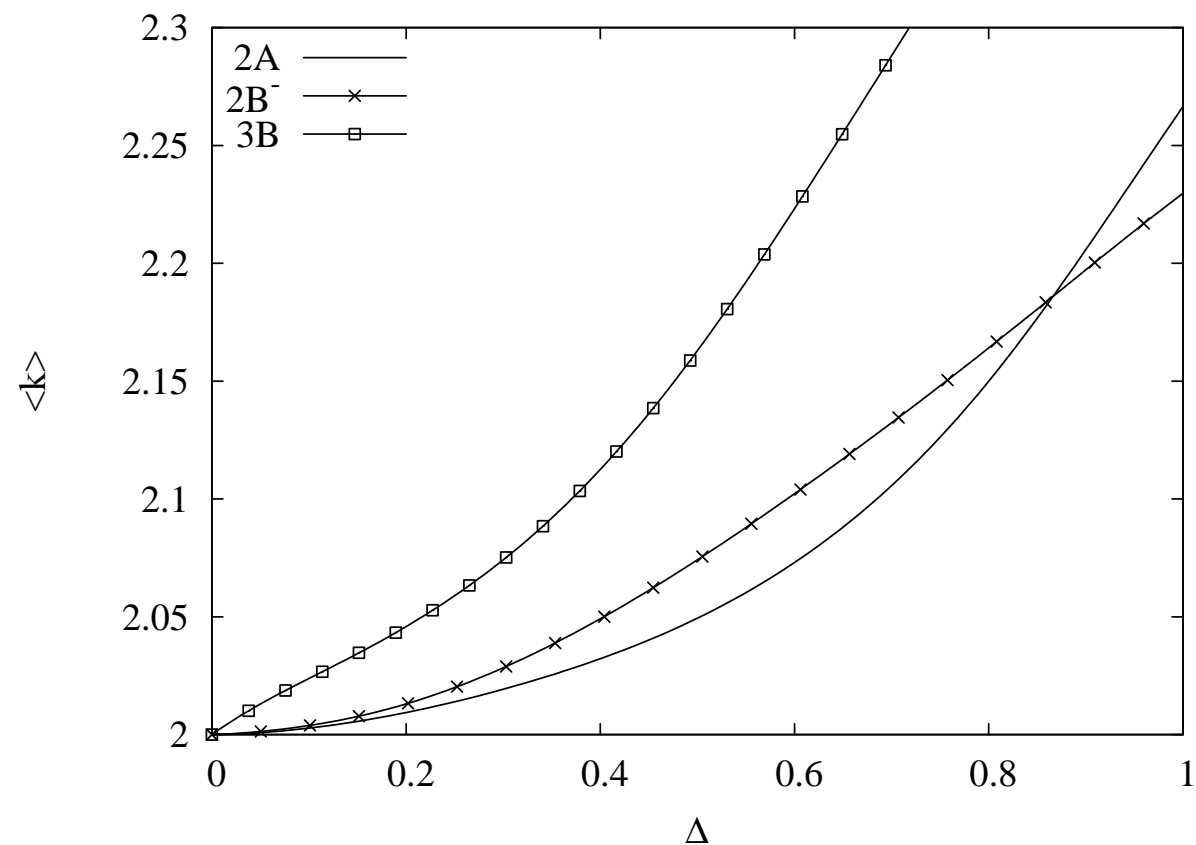

Figure 5: Specific combinations of coupling and network disorder, which leave the zero $\epsilon^{*}$ of the interaction energy independent of $\Delta$ and $\langle k\rangle$, and keep it equal to its non-perturbed value. The interaction energy has been calculated with $N=1001$ and $T=10^{6}, 10$ coupling/network disorder realizations for the $2 A$ (solid line) and $2 B^{-}$(crosses) dynamics, and 100 coupling/network disorder realizations for the $3 B$ (open squares) dynamics.

a curve $\langle k\rangle(\Delta)$ with $W\left(\epsilon^{*}\right)=0$, where $\epsilon^{*}$ is fixed to the respective value obtained for a homogeneous coupling and a unperturbed ring-network structure as in [15] (see Fig. 5). This finding implies that for these zeros the specific combination of coupling and network disorder reproduces the zero $\epsilon^{*}$ as given in [15] for the ring with homogeneous couplings.

In contrast to the zeros of the $2 A, 2 B^{-}$and $3 B$ dynamics, the zero $\epsilon_{2}^{(3 A)}$ of the $3 A$ dynamics is shifted to larger values for coupling as well as network disorder. Thus it is not possible to combine the two forms of disorder in order to maintain the value as given in [15]. In [15] the zero $\epsilon_{2}^{(3 A)}$ is related to the running electromagnetic coupling $\alpha_{e l}(E)$ at three times the electron mass scale $E=3 m_{e}$. This is a rather small energy. In fact, the fine structure constant $\alpha_{e l}(0)$ at zero energy is a lower bound for a running electromagnetic coupling. It is an interesting observation that the zero of the $3 A$ dynamics is only shifted to larger values, but not to smaller values. These would not allow for an interpretation in terms of smaller energies due to the lower bound. The larger values are associated to larger energy scales, which are of the order $10 \mathrm{MeV}$ for network disorder and of the order $10 \mathrm{GeV}$ for coupling disorder (see Fig. 6). 


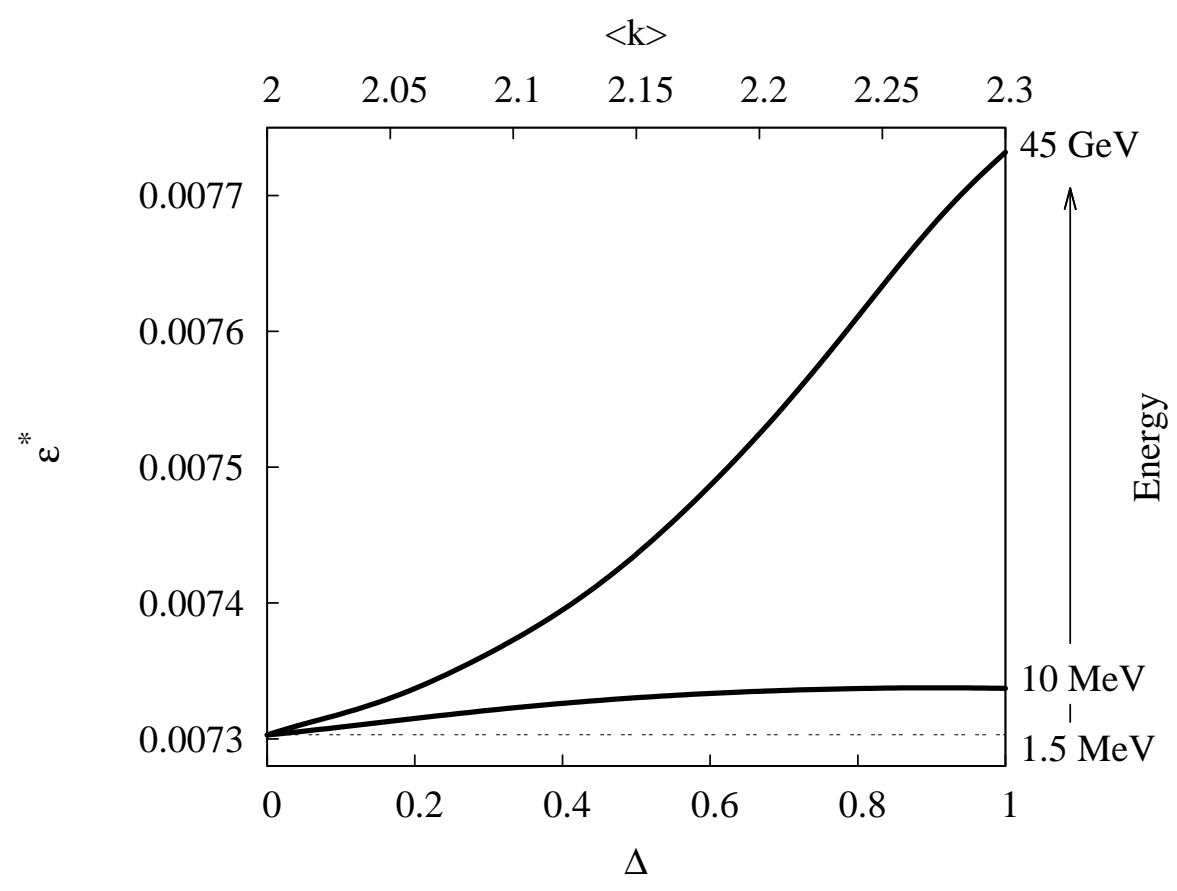

Figure 6: Energy shift in the interpretation of the zero $\epsilon_{2}^{(3 A)}$ as a running electromagnetic coupling constant $\alpha_{e l}(E)$. According to [15] the energy is $E \approx 1.5 \mathrm{MeV} \approx 3 m_{e}$ for zero coupling and network disorder. The lower curve shows the energy shift for increasing network disorder, whereas the upper curve shows the shift due to increasing coupling disorder. The running electromagnetic coupling $\alpha_{e l}(E)$ has been calculated as given in [15], with particle masses and the fine structure constant $\alpha_{e l}(0)$ taken from [18]. 


\section{Conclusion}

We have studied the effect of coupling and network disorder on the zeros of the interaction energy in chaotic strings as introduced in [15]. It has been found that certain combinations of coupling and network disorder preserve the empirical relationship between chaotic strings and the weak and strong sector of the standard model of elementary particle physics. For the electromagnetic sector it has been found that already a small disorder pushes the associated energy scale of the running coupling constant far away from the result without disorder.

Further studies on the empirical relationship between chaotic strings and the standard model of elementary particle physics should also address the more dynamical properties of chaotic strings, like for example desynchronization, synchronization and correlations in general. Although there have been extensive studies on coupled map lattices, these works do not consider the specific combinations of Tchebyscheff maps used here. The impact of coupling and network disorder as introduced in this paper deserves particular attention, since they are not only interesting in connection with the chaotic string model, but for dynamical systems in general.

\section{References}

[1] K. Kaneko, Spatiotemporal chaos in one- and two-dimensional coupled map lattices, Physica D 37 (1989) 60.

[2] K. Kaneko, Clustering, coding, switching, hierarchical ordering, and control in a network of chaotic elements, Physica D 41 (1990) 137.

[3] H. Shibata, KS entropy and mean lyapunov exponent for coupled map lattices, Physica A 292 (2001) 182.

[4] K. Kaneko, Theory and applications of coupled map lattices, John Wiley \& Sons, 1993.

[5] K. Kaneko, I. Tsuda, Complex Systems: Chaos and Beyond, Springer, 2000.

[6] M. Cross, P. Hohenberg, Pattern formation outside of equilibrium, Rev. Mod. Phys 65 (1993) 851.

[7] A. Arenas, A. Dìaz-Guilera, J. Kurths, Y. Moreno, C. Zhou, Synchronization in complex networks, Phys. Rep. 469 (2008) 93.

[8] S. Jalan, R. Amritkar, Self-organized and driven phase synchronization in coupled maps, Phys. Rev. Lett. 90 (2003) 014101.

[9] F. Atay, J. Jost, A. Wende, Delays, connection topology, and synchronization of coupled chaotic maps, Phys. Rev. Lett. 92 (2004) 144101.

[10] P. Gade, C.-K. Hu, Synchronous chaos in coupled map lattices with small-world interactions, Phys. Rev. E 62 (2000) 6409.

[11] P. Gade, C.-K. Hu, Spontaneous structure formation in a network of dynamic elements, Phys. Rev. E 67 (2003) 046226. 
[12] J. Jost, M. Joy, Spectral properties and synchronization in coupled map lattices, Phys. Rev. E 65 (2001) 016201.

[13] P. Li, M. Chen, Y. Wu, J. Kurths, Matrix-measure criterion for synchronization in coupled-map networks, Phys. Rev. E 79 (2009) 067102.

[14] C. Beck, Chaotic strings and standard model parameters, Physica D 171 (2002) 72.

[15] C. Beck, Spatio-Temporal Chaos and Vacuum Fluctuations of Quantized Fields, Vol. 21 of Advanced Series in Nonlinear Dynamics, World Scientific, 2002.

[16] C. Beck, Complexity of chaotic fields and standard model parameters, in: The Logic of Nature, Complexity and New Physics: From Quark-Gluon Plasma to Superstrings, Quantum Gravity and Beyond, Vol. 44 of The Subnuclear Series, World Scientific, 2008, pp. 1-37.

[17] J.-P. Uzan, The fundamental constants and their variation: observational and theoretical status, Rev. Mod. Phys. 75 (2003) 403.

[18] C. Amsler et al. (Particle Data Group), Review of particle physics, Physics Letters B 667 (2008) 1.

[19] K. Mönig, First bounds on the higgs boson from hadron colliders, Physics 3 (2010) 14.

[20] M. Schäfer, C. Beck, Discrete symmetries of chaotic strings, Dynamical Systems 25 (2010) 253.

[21] D. Watts, S. Strogatz, Collective dynamics of 'small-world' networks, Nature 393 (1998) 440.

[22] M. Newman, C. Moore, D. Watts, Mean-field solution of the small-world network model, Phys. Rev. Lett. 84 (2000) 3201.

[23] S. Bethke, The 2009 world average of $\alpha_{s}$, Eur. Phys. J. C 64 (2009) 689. 